\title{
Low Back Pain in Patients With Systemic Analgesic Intolerance Managed With Mesotherapy: A Case Report
}

\author{
Massimo Mammucaria, d, Enrica Maggiori ${ }^{\mathrm{b}}$, Sergio Maggiori ${ }^{\mathrm{b}}$, Alessandro F. Sabato ${ }^{\mathrm{c}}$
}

\begin{abstract}
Compounds injected by the intradermal route slowly diffuse into the underlying tissues and produce a drug-sparing effect compared to the parenteral route. This technique, called mesotherapy, can be useful when systemic drugs are not tolerated and to synergize with other therapies. We report advantages obtained in a patient with low back pain to underline that drugs introduced by intradermal route should be considered in the management of localized pain. A 52-year-old patient with low back pain was treated with analgesic drugs, but adverse events raised. After injection of intradermal drugs, pain was controlled and no adverse events were detected. Mesotherapy could represent a pharmacological strategy to manage localized pain.
\end{abstract}

Keywords: Mesotherapy; Intradermal therapy; Low back pain; Drug sparing

\section{Introduction}

We reported that compounds given in small quantities through multi-superficial punctures produce a "micro-deposits" in the dermis that slowly release into the underlying tissues [1-7]. Beyond the pharmacological effect of drugs, it was also suggested that the reflex effect produced by the needles can induce clinical benefits [8]. In fact, many studies reported pain relief in patients with several musculoskeletal or posttraumatic conditions [9]. Mesotherapy is now well

\footnotetext{
Manuscript accepted for publication March 4, 2014

aPrimary Care Unit ASL RMF, Rome, Italy

${ }^{\mathrm{b}}$ Italian Society of Mesotherapy, Rome, Italy

${ }^{c}$ Emergency Care, Critical Care Medicine, Pain Medicine and

Anesthesiology Department, University of Tor Vergata, Rome, Italy

${ }^{\mathrm{d}}$ Corresponding author: Massimo Mammucari, Primary Care Unit ASL

RMF, Rome, Italy. Email: massimo.mammucari@libero.it
}

doi: http://dx.doi.org/10.14740/jmc1743w defined as a minimally invasive technique, useful to reduce dose of active compounds, a rapid onset and a prolonged duration of action [7]. For these proprieties, the technique is applied also with vaccines [10-12] or other pharmaceutical substance $[13,14]$. We report the case of a patient who has received benefit from mesotherapy, in particular for the reduction of adverse events due to the drug-sparing effect.

\section{Case Report}

A 52-year-old European male, with no previous medical history of disease, refers to a chronic pain that increases with movement of the lumbar spine. This form of pain has been present for about 5 years and causes recurrent back pain. The painful present crisis, which led the patient to our observation, had risen 3 days before. No change in blood chemistry was found later, and the patient took the therapy prescribed by other doctors: nonsteroidal anti-inflammatory drug (NSAID) (ketoprofen $160 \mathrm{mg} /$ day i.m.), muscle relaxant (thiocolchicoside $2 \mathrm{mg}$ /day i.m.), corticosteroid (betamethasone $4 \mathrm{mg} /$ day i.m.) and a gastric protector (lansoprazol $15 \mathrm{mg} /$ day per os). After 3 days, even if the pain was reduced (NRS $=5$; evaluated with a number scale rate with 11 points, from 0 to 10 where 0 is no pain and 10 is the maximum tolerable pain), for the occurrence of gastrointestinal discomfort, his doctor suggested to stop treatment and start a therapy with opioid (codeine/acetaminophen 30/500 mg up to three times per day). Then, after a further 5 days of therapy, the patient complained of intense nausea and refused to continue the codeine/acetaminophen combination. It was suggested to rotate opioid (oxycodone $10 \mathrm{mg}$ twice a day) and metoclopramide $(10 \mathrm{mg} /$ day) and dietary rules (hydration and high-fiber diet). However, the patient, while receiving a benefit from therapy $(\mathrm{NRS}=4) 3$ days after reported nausea and gastrointestinal discomfort that prevented him from going to work and pain that prevented even the night rest.

At this time, the patient is targeted to our observation. At the medical examination, pain was localized along the lumbar paravertebral muscles (from T12 to S1) and it was also reported along the left lower limb. No signs of hyperalgesia nor allodynia were found in the lower back or along the 
course of the nerves emerging from L1 to L5. Pain at the time of the visit is reported with intensity of NRS 6. Meanwhile, magnetic resonance imaging (MRI) was performed which showed the lumbosacral interbody disc degeneration (L4L5-S1), slight protrusion of the disc (L4-L5 and L5-S1) and early interapophyseal osteoarthritis (This situation appeared to be stable compared to MRI performed 2 years before). Given the clinical and pharmacological history, we proposed to implement a local therapy with the aim of reducing the pain and the contracture of the para-vertebral muscles. We explained patient the potential benefits and limitations of mesotherapy, including potential adverse events. After a new medical examination and a new clinical anamnesis (to exclude allergies to medicines or other forms of immunological reactivity), blood pressure, heart and breathing rates were collected. It was also well-explained that the introduction of the needle and the medications on the skin surface could generate mild pain, burning or a feeling of warmth. Obtained an informed consent, we have implemented mesotherapy based on the same muscle relaxant and NSAID taken previously by parenteral route. The patient was placed in the prone position and the skin at lumbar level was thoroughly disinfected, thiocolchicoside (vial of $4 \mathrm{mg} / 2 \mathrm{~mL}$ ) was injected intradermally along the course of the para-vertebral muscles (left and right sides) and subsequently ketoprofen (vial of $160 \mathrm{mg} / 2 \mathrm{~mL}$ ) was injected along the course of the spinous processes from $\mathrm{T} 12$ to $\mathrm{S} 1$. The needle was inclined by $30^{\circ}$ with respect to the skin surface. For each micro-puncture, a minimal amount of medication was injected likely to produce a small wheal, which raised slightly the surface layer of the skin. The two drugs were prepared in two different syringes, and not mixed. During treatment with ketoprofen, the patient experienced a feeling a slight discomfort, while during the administration of thiocolchicoside, he experienced a feeling of warmth. After the treatment, only half of the vial of ketoprofen was used $(1 \mathrm{~mL})$, while the entire contents of thiocolchicoside was injected $(2 \mathrm{~mL})$. Every act was carried out with aseptic gloves, including the preparation of drugs to avoid contamination. Immediately after treatment, the patient was kept in a supine position. Blood pressure was measured again after $5 \mathrm{~min}$ and the patient was asked to sit down, and after another 5 min to get up. This waiting time has two purposes, to verify that the patient does not have a vagal syndrome that may occur after treatment with needles, and to make sure that no immune reaction was taking place. When the patient was again standing, he was asked to report back pain and the feeling was a slight discomfort at the point of inoculation, but it seemed that there was a rapid improvement in pain $(\mathrm{NRS}=3)$. At the end of the first session of treatment, we have also prescribed a low dose of oxycodone/ acetaminophen (one tablet of $5 / 325 \mathrm{mg} /$ day) to be taken in the evening before going to bed in order to reduce nighttime pain that prevented the patient to rest. The patient has carried out the same mesotherapy schedule after 3, 7 and
14 days (always with a tablet of oxycodone/acetaminophen in the evening) with an average pain recorded immediately before each session of NRS 3, 3, 2, respectively. After the fourth session (carried out after 21 days), the patient stated that the pain had disappeared, and the treatment was discontinued. Throughout the treatment period, no adverse events have been reported (apart from mild pain and the feeling of warmth at the site of intradermal injection lasting a few minutes and always resolved spontaneously). After suggesting continuing with gymnastics and regular medical visits for follow-up the vertebral situation, the case was closed. In the medical record, we reported all clinical data and the final results of this treatment.

\section{Discussion}

Some patients with chronic pain reported adverse events from systemic therapies, and others have contraindications to the use of long-term medication. NSAIDs are often contraindicated or cannot be administered for a long period due to adverse cardiovascular or kidney disease. For these reasons, it is convenient to balance the clinical efficacy and the occurrence of adverse events looking for the minimal effective and the maximum tolerated dose. In this regard, we have reported the effect of mesotherapy which reduces the required dose (and the frequency of administration) of certain analgesics, and at the same time can synergize with other therapies in the management of pain [8]. Sometimes, it is necessary to review the treatment regimen and, based on the response obtained, increase or decrease the frequency of the treatment. In the case that we presented, mesotherapy allowed to control pain, avoiding adverse effects of oral therapy, with a weekly treatment. We suggested to insert the mesotherapy in the scheme of treatment of patients who need to reduce the dosages of systemic drugs, or who cannot take them because of a risk benefit ratio unfavorable, and to get a drug-sparing effect $[7,9]$. When performing mesotherapy, attention must be paid on certain aspects. In fact, it is necessary to obtain a specific informed consent, in particular by clarifying the advantages and limitations of mesotherapy [15]. It is also important to assess the patient at regular intervals in order to assess whether the treatment plan set is suitable to the needs of the individual patient. Mesotherapy also assumes importance for several outpatients in multi-drug treatment (with an increased risk for drug-drug interactions) that needs analgesic therapy for localized pain. In conclusion, the clinician who has the knowledge of the mesotherapy technique has an additional pharmacological strategy to use, naturally when the medical need arises. Finally, but not least, we underline that the patient point of view, local therapy, when it is effective and feasible, leads to a high acceptability by the patient. In this case, pain management has allowed the normal working activity and the satisfaction of the patient. 


\section{Competing Interests}

The authors declare that they have no competing interests.

\section{Authors Contributions}

MM made substantial contributions to the conception and design of this report, drafting the manuscript and revising it critically. EM, SM and AFS reviewed the manuscript and approved it.

\section{References}

1. Binaglia L, Marconi P, Pitzurra M. The diffusion of intradermally administered procaine. J Mesother. 1981;1:1528.

2. Binaglia L, Marconi P, Pitzurra M. Absorption of $\mathrm{Na}$ ketoprofen administered intradermally. J Mesother. 1981;1:85-91.

3. Pitzurra M, Marconi P. Immunogenesis and mesotherapy: the immunoresponse to antigens inoculated intradermally. J Mesother. 1981;1:9-14.

4. Kaplan JA, Coutris G. Mesoscintigraphie et proposition dune theorie unifiee de la mesotherapie. In: Bulletin 5 des communications du 6e Congres International de Mesotherapie; 1992, Paris, Franca. p. 2-4.

5. Mrejen D. Semeiologie. Pharmacocinetique et profondeur des injections en mesotherapie. In: Bulletin 5 des communications du 6e Congres International de Mesotherapie; 1992; Bruxelas, Belgica. Paris: Societe Francaise de Mesotherapie; 1992. p. 13-14.

6. Hsu CC, Kuo HC, Hsu CT, Gu Q. Abdominal mesotherapy injection extended the absorption of follicle-stimulating hormone. Fertil Steril. 2011;95(6):2134-2136,
2136 e2131.

7. Mammucari M, Gatti A, Maggiori S, Bartoletti CA, Sabato AF. Mesotherapy, definition, rationale and clinical role: a consensus report from the Italian Society of Mesotherapy. Eur Rev Med Pharmacol Sci. 2011;15(6):682694.

8. Crenna P, Mancia P. Reflex actions in mesotherapy. J Mesother. 1981;1:29-40.

9. Mammucari M, Gatti A, Maggiori S, Sabato AF. Role of mesotherapy in musculoskeletal pain: opinions from the italian society of mesotherapy. Evid Based Complement Alternat Med. 2012;2012:436959.

10. Kenney RT, Frech SA, Muenz LR, Villar CP, Glenn GM. Dose sparing with intradermal injection of influenza vaccine. N Engl J Med. 2004;351(22):2295-2301.

11. Coudeville L, Andre P, Bailleux F, Weber F, Plotkin S. A new approach to estimate vaccine efficacy based on immunogenicity data applied to influenza vaccines administered by the intradermal or intramuscular routes. Hum Vaccin. 2010;6(10):841-848.

12. Sticchi L, Alberti M, Alicino C, Crovari P. The intradermal vaccination: past experiences and current perspectives. J Prev Med Hyg. 2010;51(1):7-14.

13. Maggiori E, Bartoletti CA, Maggiori S, Tomaselli F, Dorato D. Local intradermotherapy (ITD) with mesoglican in PEFS and CVLI, retrospective study. Trends Med. 2010;(10):73-78.

14. Maggiori E, Bartoletti E, Mammucari M. Intradermal therapy (mesotherapy) with lymdiaral in chronic venous insufficiency and associated fibrosclerotic edema damage: a pilot study. J Altern Complement Med. 2013;19(9):777-781.

15. Mammucari M, Gatti A, Maggiori E, Vergari B, Gafforio P, Tufaro G, Maggiori S. [Informed consent and experimental treatments: the case of mesotherapy]. Recenti Prog Med. 2013;104(5):214-217. 